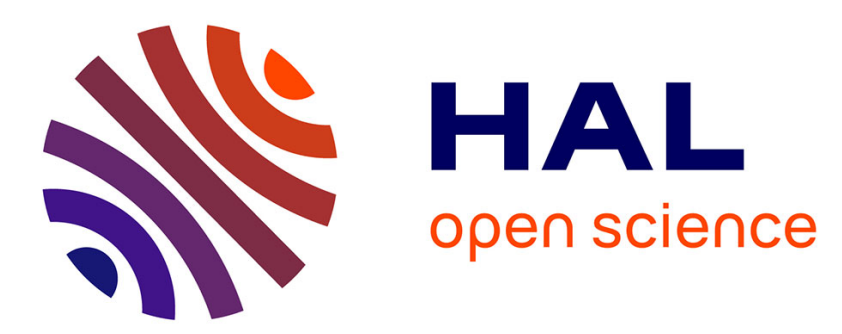

\title{
Alimentation des vaches laitières: Comparaison des différentes méthodes de prédiction des quantités ingérées
}

Philippe Faverdin

\section{To cite this version:}

Philippe Faverdin. Alimentation des vaches laitières: Comparaison des différentes méthodes de prédiction des quantités ingérées. Productions Animales, 1992, 5 (4), pp.271-282. hal-00895983

\section{HAL Id: hal-00895983 \\ https://hal.science/hal-00895983}

Submitted on 1 Jan 1992

HAL is a multi-disciplinary open access archive for the deposit and dissemination of scientific research documents, whether they are published or not. The documents may come from teaching and research institutions in France or abroad, or from public or private research centers.
L'archive ouverte pluridisciplinaire HAL, est destinée au dépôt et à la diffusion de documents scientifiques de niveau recherche, publiés ou non, émanant des établissements d'enseignement et de recherche français ou étrangers, des laboratoires publics ou privés. 
INRA Prod. Anim., 1992,5 (4), 271 - 282

\section{P. FAVERDIN}

INRA Station de Recherches sur la Vache Laitière 35590 Saint Gilles
Alimentation des vaches laitières : Comparaison des différentes méthodes de prédiction des quantités ingérées

La prédiction des quantités ingérées constitue la clé de voûte des systèmes de rationnement, en particulier pour les vaches laitières dont les variations d'appétit sont très importantes. Malgré ce rôle central de l'ingestion dans la qualité d'un rationnement, les études sur l'appétit sont peu nombreuses en comparaison des études de digestion, ce qui explique en partie le peu de renouveau des méthodes de prévision de l'ingestion. Les équations publiées pour prédire les quantités ingérées sont cependant assez nombreuses. Les rassembler et les comparer n'est pas toujours simple compte tenu des approches et des objectifs différents de leurs auteurs.

Des revues très différentes ont déjà été consacrées à la comparaison des méthodes de prédiction des quantités ingérées par les vaches laitières en privilégiant les différents facteurs de variation (Bines 1979, Wangsness et Muller 1981), la validation de quelques pré-

\section{Résumé}

Les différentes méthodes de prédiction des quantités ingérées peuvent être regroupées en 3 grands types : 1) les équations de régression multiple, généralement empiriques et issues de compilation de données expérimentales, 2) le système de 2 prédictions, l'une liée à une régulation de l'appétit de type physique (encombrement du tractus digestif), l'autre de type énergétique (satisfaction des besoins énergétiques) dont seule la plus faible est conservée pour la prédiction des quantités ingérées, 3) le système basé sur les unités d'encombrement (UE) des fourrages et la substitution de l'aliment concentré. La comparaison de ces différents systèmes montre que les facteurs les plus difficiles à prendre en compte restent d'une part la diversité des aliments et leur proportion respective dans la ration, d'autre part le début de lactation.

La plupart de ces systèmes sont plus destinés à établir des calculs de ration à l'optimum des recommandations alimentaires qu'à réellement prédire des quantités ingérées en prenant en compte tous les facteurs de variation possibles. Ils sont étroitement liés à des pratiques alimentaires (rations complètes riches en sous-produits et aliments concentrés aux USA, ajustement individualisé des apports de concentrés avec des rations majoritairement à base de fourrages en Europe). Le système des UE proposé par I'INRA présente quelques limites dans la prédiction de la valeur UE des aliments (séparation fourrages-concentrés, nombreuses équations) et dans son utilisation en début de lactation. Actuellement, il constitue cependant l'outil permettant de décrire la plus grande diversité de situations tout en conservant une méthode de calcul facile à utiliser en pratique et des possibilités d'évolution. dictions (Neal et al 1984), la diversité des rations (Waldo 1986), la présentation d'un système (Jarrige 1986, Kristensen et Ingvartsen 1986) ou encore l'approche dynamique et la modélisation (Forbes 1983, 1986).

Cette revue a pour objectif d'essayer de recenser, sans être exhaustive, les principales méthodes de prévision de l'ingestion proposées actuellement en pratique pour les vaches laitières. Ces méthodes seront comparées en analysant successivement leur aptitude à décrire les grands facteurs de variation connus et leurs possibilités d'utilisation dans des pratiques de rationnement des vaches laitières.

\section{1 / Les différentes méthodes de prédiction des quantités ingérées}

Depuis 25 ans, de nombreuses équations ont été proposées pour prédire les quantités ingérées par les vaches laitières. Toutes ces équations peuvent en fait être classées en trois groupes représentant les principales méthodes de prédiction utilisées. Les principales équations sont présentées dans le tableau 1 avec une notation commune, mais cette liste ne se veut pas exhaustive. Tous les résultats et les équations de prédictions seront exprimés en $\mathrm{kg}$ de matière sèche par animal et par jour 
Tableau 1. Les différentes méthodes de prédiction des quantités ingérées. (NB : cette liste d'équations illustre les principales méthodes utilisées mais n'est pas exhaustive).

\begin{tabular}{|c|c|c|c|}
\hline \multicolumn{3}{|c|}{ Variables prédictrices } & \multirow{2}{*}{$\begin{array}{l}\text { Aliment concentré } \\
\text { Quantité d'aliment concentré (kg MS/j) }\end{array}$} \\
\hline PV & Poids vif $(\mathrm{kg})$ & $\mathrm{C}$ & \\
\hline PL & Production de lait $(\mathrm{kg} / \mathrm{j})$ & NDFC & Teneur en NDF de l'aliment concentré (en \\
\hline TB & Taux butyreux $(\mathrm{g} / \mathrm{kg})$ & \multirow{3}{*}{ TMOC } & $\% \mathrm{MS})$ \\
\hline PL4\% & Production de lait corrigée à $4 \%$ de matières grasses $(\mathrm{kg} / \mathrm{j})$ & & Teneur en matière organique de l'aliment \\
\hline PLMAX & Production de lait maximum au cours de la lactation $(\mathrm{kg} / \mathrm{j})$ & & concentré (en \% MS)) \\
\hline PLTOT & Potentiel de production laitière par lactation $(\mathrm{kg})$ & \multirow[t]{2}{*}{$\mathrm{ENC}$} & Valeur en énergie nette de l'aliment \\
\hline VPV & Variation de poids vif $(\mathrm{kg} / \mathrm{j})$ & & concentré (en Mcal/kg MS) \\
\hline PVVEL & Poids vif juste après vêlage $(\mathrm{kg})$ & \multirow[t]{5}{*}{ VEC } & Valeur d'encombrement du concentré \\
\hline SL & Stade de lactation (en semaine/vêlage) & & \\
\hline FML & Facteur de correction pour le mois de lactation & & \\
\hline FPM & Primipares $=0$, multipares $=1$ & & Ration comnlète \\
\hline A & Age (en années) & & sation comprete \\
\hline \multirow[t]{2}{*}{ NL } & Numéro de lactation & $\mathrm{CR}$ & $\begin{array}{l}\text { Pourcentage d'aliment concentré dans la } \\
\text { ration (en } \% \text { MS) }\end{array}$ \\
\hline & Fourrage & dMSR & Digestibilité de la matière sèche de la ration \\
\hline $\mathrm{F}$ & Quantité de fourrage ingérée $(\mathrm{kg} \mathrm{MS} / \mathrm{j})$ & NDFR & Teneur en NDF de la ration (en $\%$ MS) \\
\hline TMSF & Teneur en matière sèche (en \%) & \multirow[t]{6}{*}{ DER } & \multirow{5}{*}{$\begin{array}{l}\text { Densité énergétique de la ration (en Mcal } \\
\text { d'énergie digestible }\end{array}$} \\
\hline TMOF & Teneur en matière organique (en \% MS) & & \\
\hline $\mathrm{dMSF}$ & Digestibilité de la matière sèche & & \\
\hline $\mathrm{dMOF}$ & Digestibilité de la matière organique & & \\
\hline q & Rendement de l'énergie métabolisable & & \\
\hline $\mathrm{CBF}$ & Teneur en cellulose brute (en \% MS) & & Environnement \\
\hline NDFF & Teneur en NDF (en \% MS) & TEMP & Température $\left(\mathrm{en}^{\circ} \mathrm{C}\right)$ \\
\hline $\mathrm{ADFF}$ & Teneur en ADF (en \% MS) & BOUE & Coefficient correcteur en fonction de la \\
\hline NNH3F & Azote ammoniacal de l'ensilage ( $\mathrm{g} / \mathrm{kg} \mathrm{N}$ total) & & portance du sol \\
\hline ENF & Valeur en énergie nette du fourrage (en Mcal/kg MS) & \multirow[t]{2}{*}{ SAISON } & Coefficient correcteur en fonction de la \\
\hline UFF & Valeur énergétique du fourrage en UFL & & saison \\
\hline VEF & Valeur d'encombrement du fourrage & & \\
\hline LEG & Proportion de légumineuse dans la composition floristique c & $\%)$ & \\
\hline HAL & Quantité d'herbe allouée (kg MO/Vache/j) & & \\
\hline BIOM & Biomasse présente dans la pâture (en $\mathrm{kg} \mathrm{MO} / \mathrm{ha}$ ) & & \\
\hline
\end{tabular}

\section{Equations de prédiction}

\section{a/ Régression multiple}

Référence

MAFF (1975)

Neal et al (1984)

Journet et al (1965)

$\operatorname{ARC}(1980)$

Bines (1979)

McCullough (1981)

Curran et al (1970)

McCullough (1973)

Vadiveloo et al (1979)

Yungblut et al (1981)

Greenhalgh et al (1966)

Curran et Holmes (1970)

Meijs et Hoekstra (1984)

Caird et Holmes (1986)
Equation de prédiction de la matière sèche totale ingérée $(\mathrm{kg} / \mathrm{j})$

$0.025 * \mathrm{PV}+0.1 * \mathrm{PL}$

$0.022 * \mathrm{PV}+0.2 * \mathrm{PL}$

$6.7+0.007 * \mathrm{PV}+0.27 * \mathrm{PL}$

$\left(0.135 * \mathrm{PV} 0.75+0.2 *\left(\mathrm{PL}-21.4 * \mathrm{SL}^{0.2 *} \exp (-0.04 * \mathrm{SL})\right)\right) * \mathrm{CF} * \mathrm{COR}$

avec $\mathrm{CF}=(0.81,0.98,1.07,1.08,1.09,1.08,1.01,0.99,0.97,0.93)$ respectivement pour les mois 1 à 10 de lactation si $\mathrm{q}<0.55, \mathrm{COR}=(1-(0.55-\mathrm{q}) * 3)$ sinon $\mathrm{COR}=1$

$(1+0.2 * \mathrm{FPM}) *\left(4.25+0.0113 * \mathrm{PV}+0.16 * \mathrm{PL}+2.45^{*} \mathrm{VPV}\right)$

$-5.54+0.022 * \mathrm{PV}+0.511 * \mathrm{PL} 4 \%-0.0024 * \mathrm{PL} 4 \%{ }^{2}$

$(-3.28+0.66 * \mathrm{C}+0.18 .4 * \mathrm{dMOF}+0.094 * \mathrm{PL}) * 100 / \mathrm{TMOF} \quad$ en milieu de lactation

$5.38+0.008 * \mathrm{PV}+0.359 * \mathrm{PL} 4 \%-0.028 * \mathrm{CR}$

$0.076+0.404 * \mathrm{C}+0.015 * \mathrm{PV}-0.129 * \mathrm{SL}+4.12 * \log (\mathrm{SL})+0.14 * \mathrm{PL}$

$3.37+0.01 * \mathrm{PV}+0.34 * \mathrm{PL}+0.053 * \mathrm{~TB}+0.34 * \mathrm{NL}-0.11 * \mathrm{ADF}$

Equations calculée au pâturage

$\left(0.055^{*} \mathrm{PV}^{0.75}+0.20 * \mathrm{PL} 4 \%+1.03 * \mathrm{VPV}\right) * 100 /(\mathrm{dMOF} * \mathrm{TMOF})$

$(-1.63+0.129 * \mathrm{PV} 0.75+0.22 * \mathrm{PL}-0.49 * \mathrm{~A}) * 100 / \mathrm{TMOF}$

$\left(-0.6+0.981 * \mathrm{HAL}-0.014 * \mathrm{HAL}^{2}+1.479 * \mathrm{C}-0.039 * \mathrm{C} * \mathrm{HAL}\right) * 100 / \mathrm{TMOF}$

$\left(0.323+0.177 * \mathrm{PL}+0.010 * \mathrm{PV}+1.636 * \mathrm{C}-1.008 * \mathrm{BIOM}+0.54 * \mathrm{HAL}-0.006 * \mathrm{HAL}^{2}-0.048 * \mathrm{HAL}^{*} \mathrm{C}\right) * 100 / \mathrm{TMOF}$

(équation pour pâturage tournant)

$\mathrm{PV} * 0.7 * 0.024 *\left(1+0.0656^{*} \mathrm{SL}^{1.7 *} \exp (-0.147 * \mathrm{SL})+0.013 *(\mathrm{PLMAX}-\mathrm{PV} / 20)\right) *(1-1.7 *(0.8-\mathrm{dMSF})+0.0017 * \mathrm{LEG}) * \mathrm{CT}$ avec $\mathrm{CT}=1$ si $\mathrm{TEMP}<=25$ et $\mathrm{CT}=1-0.02 *(\mathrm{TEMP}-25)$ 
Brown et al (1977)

Cornell Fox et al (1990) $\exp (0.52+$ SAISON-0.0058*SL+0.148*LN(7*SL)+0.339*LN(PL)+0.0000993*PL*TB+0.000675*PV+ $\left.0.018 * \mathrm{CB}-0.000557 * \mathrm{CB}^{2}\right)$

avec SAISON=(-0.041 automne hiver, 0.022 printemps, 0.019 été $)$

$(0.0185 * \mathrm{PV}+0.305 * \mathrm{PL} 4 \%) * \mathrm{CT} 1 * \mathrm{BOUE}$

$\mathrm{CT} 1=1$ entre 15 et $25^{\circ} \mathrm{C}, \mathrm{CT} 1=0.9$ entre 25 et $35^{\circ} \mathrm{C}$ et plus si nuit fraiche, $\mathrm{CT} 1=0.65$ si $>35^{\circ} \mathrm{C}$ et nuit chaude $\mathrm{CT} 1=1.03$ entre 5 et $15^{\circ} \mathrm{C}, 1.05$ entre -5 et $5^{\circ} \mathrm{C}, 1.07$ entre -15 et $-5^{\circ} \mathrm{C}$ BOUE $=0.85$ si 10 à $20 \mathrm{~cm}, 0.7$ au delà

\section{b/ Prédiction "physique" et "énergétique"}

Dans ce système de prédiction proposé par Conrad (1966), on calcule 2 équations de prédiction des quantités ingérées limitées par l'énergie (E) ou par l'encombrement physique du tractus digestif $(\mathrm{P})$ et on retient la plus faible valeur

Forbes (1977)

Ce modèle reprend les équations de Conrad (1966) en intégrant les variations de volume du rumen

$\mathrm{E}=0.45 * 10^{\wedge}(0.55-0.46 * \log (\mathrm{dMSR} * 100)+0.51 * \log (2.2 * \mathrm{PV})+0.25 * \log (0.73 * \mathrm{PL} 4 \%+5 * \mathrm{VPV}))$

$\mathrm{P}=0.45 * 10^{\wedge}(1.53 * \log (\mathrm{dMSR} * 100)+1.01 * \log (2.2 * \mathrm{R})+0.99 * \log (2.2 * \mathrm{PV})-5.3)$

avec $\mathrm{R}=4 *(\mathrm{PVVEL} / 5-(\mathrm{PV}-\mathrm{PVVEL}) / 3) /(\mathrm{PVVEL} / 5)$

Mertens (1987)

$\mathrm{E}=(0.08 * \mathrm{PV} 0.75+0.74 * \mathrm{PL} 4 \%-4.92 * \mathrm{VPV}(<0)+5.12 * \mathrm{VPV}(>0)) /((\mathrm{C} * \mathrm{ENC}+(100-\mathrm{C}) * \mathrm{ENF}) / 100)$

$\mathrm{P}=1.2 * \mathrm{PV} /(\mathrm{CR} * \mathrm{NDFC}+(100-\mathrm{CR}) * \mathrm{NDFF}) / 100)$

NRC (1987)

$E=\left(0.453 * P^{0.593 * P L 4 \% 0.33 *} \exp (0.16 * \mathrm{VPV})\right) / \mathrm{DER}$

$\mathrm{P}=1.1 * \mathrm{PV} /(100-\mathrm{dMSR} * 100)$

si SL $<4, \mathrm{MST}=\mathrm{MST} * 0.82$

\section{c/ Encombrement du fourrage et substitution de l'aliment concentré}

$\mathrm{CI}=$ Capacité d'ingestion de l'animal

Dans les 3 systèmes d'unités d'encombrement HYPPO, INRA et DANOIS, le principe est identique :

La somme des encombrements pondérés des fourrages et des concentrés doit être égal à la capacité d'ingestion

$\mathrm{CI}=\mathrm{F}^{*} \mathrm{VEF}+\mathrm{C}^{*} \mathrm{VEC}$

Hyppölä et Hasunen (1970)

$\mathrm{CI}=0.109 * \mathrm{PV}^{0.75}$

VEF

Racines et tubercules $\quad 0.6$

Feuilles et collets de betteraves $\quad 0.8$

Fourrage entier $\quad 1.0$

$\mathrm{VECH}=0.5$

INRA - Dulphy et al (1987)

$\mathrm{CI}=(1-0.1 *(1-\mathrm{FMP}))^{*}(22-8.25 * \exp (-0.02 * \mathrm{PL} 4 \%)+0.01 *(\mathrm{PV}-600))$

VEF environ 1000 valeurs d'aliments différents présentées dans des tables ou logiciel $\mathrm{VEC}=\mathrm{VEF} * 1.2 * \mathrm{PL} 4 \%(-0.69) * \exp \left(1.46^{*} \mathrm{UFF} / \mathrm{VEF}\right)$

(NB: la formule de calcul de VEC est la formule simplifiée qui n'est acceptable que lorsque le bilan énergétique est n'est pas trop différent de 0))

Kristensen et Ingvartsen

Primipares : $\mathrm{CI}=5.28+(\mathrm{PLTOT}-6500) / 1000 * 0.2+0.006 *(\mathrm{PV}-500)-2.22 * \exp (-0.28 * \mathrm{SL})$

Multipares : $\mathrm{CI}=6.48+($ PLTOT -6500$) / 1000 * 0.2+0.006 *(\mathrm{PV}-575)-2.69 * \exp (-0.42 * \mathrm{SL})$

$\begin{array}{ll}\text { valeurs exemples } & \text { VEF } \\ \text { Betterave fourragère } & 0.25 \\ \text { Ensilage de mais } & 0.40 \\ \text { Ensilage d'herbe (dMO=0.8) } & 0.45 \\ \text { Ensilage d'herbe }(\mathrm{dMO}=0.7) & 0.50 \\ \text { Ensilage d'herbe (dMO=0.6) } & 0.55 \\ \text { Paille d'orge } & 0.85\end{array}$

$\mathrm{VEC}=0.22 \quad$ (sauf pulpe de betterave déshydratée : $\mathrm{VEC}=0.20$ )

Le système d'équation de Lewis n'est pas vraiment un système d'encombrement, mais il est est très proche

1) Estimation de l'ingestibilité du fourrage seul (en $\mathrm{g} / \mathrm{kg} \mathrm{PV}^{0.75}$ )(ensilage d'herbe seulement $\mathrm{IF}=45+1.03 * \mathrm{TMSF}+51.6 * \mathrm{dMOF}-0.05 * \mathrm{NNH} 3$

2) Calcul de la MST ingérée en intégrant la substitution par l'aliment concentré $\mathrm{MST}=\mathrm{PV} 0.75 *\left(1.068 * \mathrm{IF}-2.47 * \mathrm{IF}^{*} \mathrm{C} / \mathrm{PV} 0.75-3370 *(\mathrm{C} / \mathrm{PV} 0.75)^{2}+0.00175 * \mathrm{PL}^{2}-10.9\right) / 1000+\mathrm{C}$ 
(kg MS) afin de pouvoir effectuer des comparaisons. En effet, de nombreux auteurs utilisent des équations pour prédire la matière organique ingérée ou la matière organique digestible ingérée et expriment leurs variables en $\mathrm{kg}$ ou en lb pour les données anglo-saxones anciennes.

\section{1 / Les régressions multiples "empiriques"}

La plupart des équations utilisées pour la prédiction des quantités ingérées sont obtenues par des ajustements de modèles statistiques (régressions multiples) à des données expérimentales. Dans ce groupe ont été rassemblées les équations de prédiction des quantités ingérées qui rassemblent en une équation empirique toutes les variables descriptives de la prédiction. Ces équations sont assez nombreuses et proviennent généralement d'une compilation de multiples essais dans lesquels des vaches recoivent tout ou partie de la ration à volonté. Suivant la nature des variables prédictrices utilisées dans ces régressions multiples, il est possible de distinguer 3 groupes : les équations qui n'utilisent que des variables descriptives de l'animal, celles qui introduisent également des éléments descriptifs de la ration, et enfin celles qui ajoutent des paramètres d'environnement.

\section{a / Equations n'utilisant que les caractéristiques de l'animal}

Ces équations sont assez nombreuses et utilisent les variables de production laitière (PL), de production de lait 4\% (PL4\%), de taux butyreux (TB), de poids vif (PV), de variation de poids vif (VPV), de stade de lactation (SL) exprimé en jour, semaine ou mois et enfin d'âge (primipare-multipare ou âge en années suivant les auteurs). Les équations qui sont présentées sont celles de Journet et al (1965), du MAFF (1975) et de l'ARC (1980), de Bines (1979), de McCullough (1981) et de Neal (1984). Certaines comme celles du MAFF (1975) et de Neal (1984) sont suffisamment simples pour être résolues par un calcul mental.

\section{b / Equations utilisant des caractéristiques de l'animal et de la ration}

Ces équations utilisent les mêmes variables de description des caractéristiques de l'animal, mais introduisent des informations sur la composition de la ration. La donnée la plus fréquemment utilisée est la quantité d'aliments concentrés $(\mathrm{C})$ ou sa proportion (CR) dans la ration. On trouve également des données de la composition chimique ou de la valeur alimentaire de la ration ou du fourrage suivant l'importance de ce dernier dans la composition des rations utilisées. Dans les équations retenues, citons la teneur en $\mathrm{ADF}$ de la ration (ADFR) ou la teneur en cellulose brute du fourrage (CBF), la digestibilité de la matière organique du fourrage (dMOF) ou de la matière sèche (dMSF), la teneur en matière organique (TMOF).

L'estimation des quantités ingérées au pâturage a conduit certains auteurs à introduire des données concernant les caractéristiques de la conduite au pâturage comme la biomasse présente par hectare (BIOM), la quantité d'herbe allouée par animal et par jour (HAL) ou encore les caractéristiques du couvert végétal comme la proportion de légumineuses dans l'herbe pâturée (LEG).

\section{c / Equations utilisant des caractéristiques de l'animal, de la ration et de l'environnement}

Ce type d'information est le plus souvent introduit sous forme de constante multiplicative visant à corriger l'ingestion moyenne en fonction des conditions climatiques. L'effet de la température est le plus connu (TEMP) mais il peut être corrigé lorsque les intempéries sont importantes et dégradent les conditions de l'environnement. Le modèle du centre de Cornell aux USA (Fox et al 1990) a ainsi introduit un terme correctif fonction de la portance de terrain (épaisseur de boue, BOUE) suite à des pluies importantes et qui traduit l'état d'inconfort des animaux dans ces conditions climatiques difficiles (pluies, vent,...). Seule l'équation de Brown et al (1977) introduit un facteur saison dans l'équation sous forme d'une constante additive propre à chaque saison.

\section{2 / Système de deux prédictions basées sur la régulation, l'une physique et l'autre énergétique, de l'ingestion}

Ces méthodes sont toutes dérivées du système d'équations proposé par Conrad et al (1966). Dans ce système, deux équations sont calculées simultanément pour prédire l'ingestion (figure 1). La première suppose que l'animal est essentiellement soumis à une régulation de type énergétique et que les quantités ingérées permettront de satisfaire le besoin énergétique de l'animal. La seconde équation suppose que l'ingestion de l'animal est limitée par l'encombrement du tractus digestif par la ration. Cette équation fait l'hypothèse que le contenu digestif ne peut contenir une masse indigestible (ou lentement digestible) supérieure à une certaine fraction du poids vif de l'animal. La valeur prédite la plus faible est retenue comme estimation des quantités ingérées, l'objectif idéal de rationnement étant théoriquement situé au point de rencontre des 2 équations. Cette méthode de prédiction ne considère la ration que complète et ne sépare pas les différents constituants. Par contre, elle ne nécessite aucune distinction à priori entre fourrages et aliments concentrés. 
Les équations de Conrad et al (1966), basées sur une limitation physique de l'ingestion par la fraction indigestible de la ration, ont été reprises par Forbes (1977) comme base pour simuler les variations de quantités ingérées au cours du cycle de lactation. L'originalité de ce modèle consiste à approcher de facon dynamique l'évolution de la capacité d'ingestion au cours du cycle lactation-gestation en prenant notamment en compte les problèmes d'état d'engraissement et leurs conséquences sur le volume abdominal. Le système du NRC est très voisin des 2 équations de Conrad et al (1966), l'équation énergétique ayant été modifiée suite à un travail plus récent de Owdongo et Conrad (1983). Enfin, le système de Mertens (1987) reprend le même principe mais en indexant la limitation physique de l'ingestion sur le contenu en NDF de la ration, en fonction du poids vif de l'animal.

Dans ce type de système, l'animal ne peut théoriquement pas consommer plus d'énergie que ses besoins, lesquels incluent la gestion des réserves corporelles, alors qu'une vache peut consommer plus et engraisser fortement avec des rations très palatables (en particulier lorsqu'elle est tarie). De plus, le contenu indigestible ne doit pas varier avec la qualité des rations lorsque l'animal est théoriquement limité par l'encombrement, ce qui ne traduit pas les observations effectuées sur bovins (Dulphy et Faverdin 1987). Il existe donc avec cette méthode de prédiction des biais intrinsèques susceptibles de diminuer la fiabilité des résultats.

\section{3 / L'encombrement des fourrages et la substitution de l'aliment concentré}

Comme dans les systèmes précédents, ce type de prédiction suppose que l'encombrement du tractus digestif, et en particulier du rumen, par les fourrages, limite leur ingestion. C'est à partir de cette constatation expérimentale que Hyppölä et Hasunen (1970) ont proposé un premier système basé sur une nouvelle unité, l'unité d'encombrement (UE). C'est dans cette unité que sont exprimés la capacité d'ingestion de l'animal (CI) et l'encombrement des différents constituants de la ration (Valeur d'encombrement des fourrages VEF, des aliments concentrés VEC). De ce fait, la CI peut être estimée indépendamment de la composition de la ration et être calculée à partir de variables propres à l'animal. Ce système est additif comme pour les apports d'énergie ou de protéines. Si un des aliments est distribué à volonté dans une ration, la somme des différents constituants de la ration, pondérée par leur encombrement, doit satisfaire la CI de l'animal exprimée en UE. Cependant, le système proposé par Hyppölä considère que la CI des vaches laitières ne varie pas avec leur niveau de production, ce qui limite les applica-

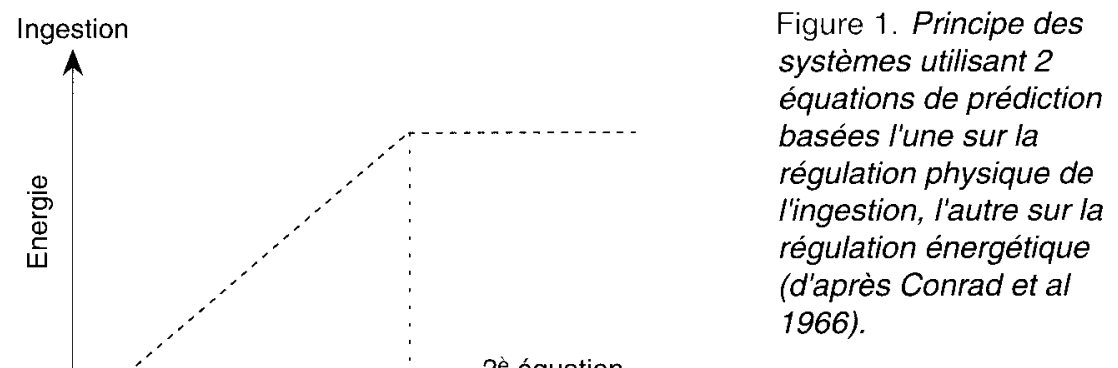
systèmes utilisant 2 équations de prédiction asées l'une sur la régulation physique de d'après Conrad et al 1966).

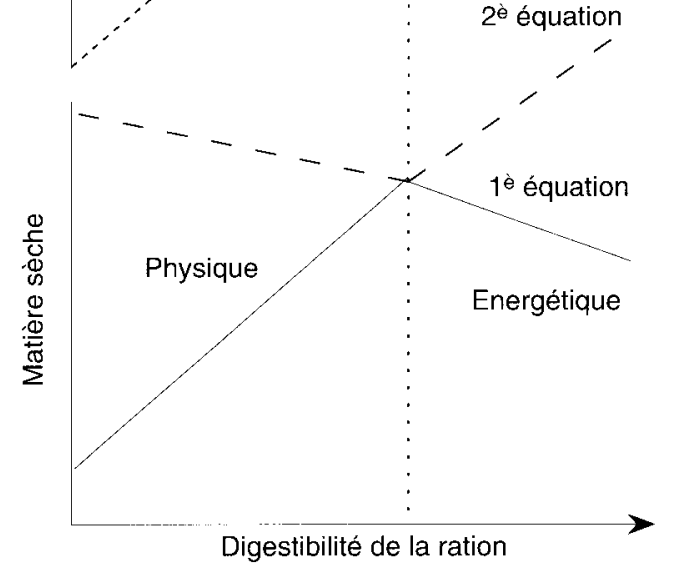

tions pratiques de ce système. En 1978, Jarrige et al ont proposé un système des UE (INRA 1978) plus complet avec :

- un nombre très élevé de données utilisées (2400 échantillons d'aliments) pour estimer la valeur d'encombrement des fourrages (Demarquilly et al, in INRA 1978)

- une CI des vaches laitières prenant en compte la production laitière

- une valeur d'encombrement des aliments concentrés qui n'est pas fixe, mais proportionnelle au taux de substitution du fourrage au concentré. Dans la version 1987 du système, ces phénomènes de substitution ont été mieux décrits grâce à des essais récents (Faverdin $e t$ al 1992). Ils ont été modélisés en intégrant le fait que les taux de substitution augmentent lorsque le bilan énergétique s'accroît (Dulphy et al 1987). Cela permet de donner une précision plus grande dans le calcul des rations de plus en plus riches en aliments concentrés du fait de l'augmentation du niveau de production laitière des animaux. Pour faire face à une méthode de calcul plus complexe avec ce nouveau modèle de substitution, une équation simplifiée et des tables (valables exclusivement en lactation et dans des situations où la vache n'est pas trop sous-alimentée) ont été proposées pour l'utilisation pratique. De plus, un logiciel, INRAtion, a été développé et permet d'utiliser toutes les possibilités du système.

Les danois (Kristensen et Ingvartsen 1986) ont proposé un système très voisin du système d'Hyppölä et Hasunen (1970) mais dans lequel l'estimation de la CI des vaches a été considérablement améliorée.

Enfin, il convient de citer le système d'équations de Lewis (1981), pour des rations à base 
d'ensilage d'herbe, dont la structure n'est pas très différente dans le principe du système des UE. La première équation permet de calculer l'ingestibilité du fourrage seul (l'inverse de son encombrement) et la seconde permet de calculer les phénomènes de substitution liés à l'apport d'aliments concentrés.

Une limite intrinsèque de ce type de système par rapport au précédent repose sur la séparation faite entre fourrages et aliments concentrés. Si cette séparation semble généralement évidente et cohérente par rapport aux pratiques d'alimentation rencontrées en Europe, des situations litigieuses peuvent perturber l'utilisateur et le calcul des rations. Ainsi le maïs plante entière est considéré comme un fourrage lorsqu'il est sous forme d'ensilage alors qu'il contient une proportion importante de grains, mais lorsqu'il est déshydraté et présenté en gros bouchon il devient un concentré. D'autres exemples montreraient que cette séparation est floue pour tous les sous-produits ainsi que pour des fourrages soumis à des traitements technologiques. Ceci ne constitue cependant un réel problème que lorsque les aliments dont la classification est ambiguë sont incorporés en quantité importante dans la ration.

Cette présentation rapide des principaux systèmes de prédiction des quantités ingérées actuellement proposés montre déjà qu'ils comportent, de par leur structure, un certain nombre de limites. Pour juger de l'importance de celles-ci, il est intéressant de voir dans quelle mesure les prédictions issues de ces systèmes sont capables de décrire les grands facteurs de variations des quantités ingérées.

Figure 2. Simulation de l'effet du poids vif des vaches laitières sur la prédiction des quantités ingérées d'une ration complète composée d'ensilage de maïs (70\%) et d'aliments concentrés (30\%)

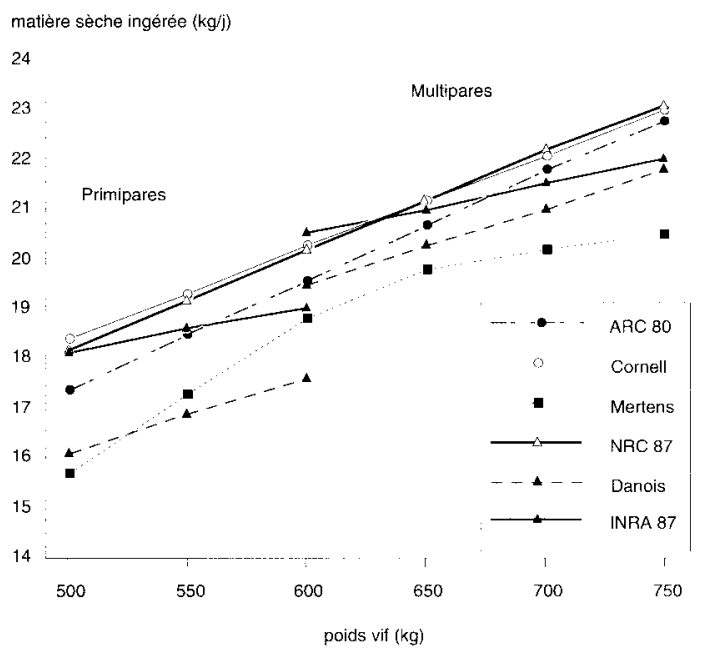

\section{2 / Les différents facteurs de variation des quantités ingérées et leur prédiction par les systèmes}

Les caractéristiques des races laitières et des régimes utilisés dans les pays où des systèmes de prédiction des quantités ingérées ont été développés sont très différentes. Pour être objectif, il convient donc de juger la qualité de la prédiction des différents systèmes plus dans l'aptitude à décrire globalement les grands facteurs de variations que dans la précision des résultats (qui peut dépendre du choix des exemples retenus). Les différences importantes de prédiction des quantités ingérées qui pourront apparaître dans les exemples qui vont suivre ne remettent pas forcement en cause la qualité des systèmes. Elles montrent cependant qu'il est souvent difficile d'adapter à nos pratiques un système de prédiction établi à l'étranger dans des conditions différentes de celles rencontrées en France. Les systèmes retenus pour les comparaisons qui suivent ont été choisis en fonction de leur représentativité et de leur actualité.

\section{1 / Animal}

\section{a / Poids vif}

Le coefficient utilisé avec la variable de poids vif de l'animal varie très largement entre les différents systèmes (de 0,7 à $2,2 \mathrm{~kg} \mathrm{MS} / \mathrm{kg}$ de poids vif). La simulation effectuée avec une ration complète à base d'ensilage de maïs $(70 \%)$ et d'aliments concentrés $(30 \%)$ permet de mieux comprendre ces différences (figure 2). Les 2 systèmes (Danois et INRA) qui présentent les coefficients les plus faibles sont ceux qui introduisent un facteur primipares-multipares dans la prédiction. Les autres équations (ARC, NRC et Cornell) qui ne prennent pas en compte l'âge des animaux utilisent un coefficient plus élevé qui traduit essentiellement les variations moyennes entre primipares et multipares mais qui ne reflète pas obligatoirement l'effet du poids vif pour des animaux de même parité. L'effet du format observé au sein d'animaux d'une même classe d'âge (Faverdin et al 1987) semble d'ailleurs assez en accord avec un coefficient modéré entre le poids vif et la MST. Il n'est cependant pas exclu que l'effet du poids vif sur les quantités ingérées soit variable suivant le type de régime utilisé. C'est ce que propose le système de Mertens (1987) qui revient à utiliser un coefficient très élevé lorsque la limitation physique est importante et faible lorsque les besoins énergétiques sont satisfaits.

\section{b / Production laitière}

Comme pour le poids vif, les coefficients rencontrés dans les différentes équations sont très variables. Dans la simulation présentée sur la figure 3 , les animaux reçoivent une ration complète à base d'ensilage de maïs 
$(70 \%)$ et d'aliments concentrés $(30 \%)$. Les systèmes de l'ARC, du NRC et de l'INRA conduisent à des prédictions de quantités ingérées très voisines. Le système de Cornell prédit un accroissement des quantités ingérées plus important sous l'effet de la production laitière car il n'intègre pas la composition de la ration dans son calcul. De ce fait, les animaux produisant généralement $45 \mathrm{~kg}$ de lait reçoivent en moyenne une ration avec plus de $30 \%$ d'aliments concentrés qui est sans doute plus ingestible. Le système danois présente une faible augmentation de la capacité d'ingestion dans cette situation car le taux de substitution de l'aliment concentré est constant et, en particulier, ne varie pas avec le bilan énergétique de l'animal. Enfin le système de Mertens (1987) montre les défauts de l'équation physique de prédiction des quantités ingérées qui, compte tenu de la teneur en NDF de la ration, bloque la prédiction de la MST à $20 \mathrm{~kg}$. Il est certain que l'animal accroît son contenu en NDF au fur et à mesure que son déficit énergétique augmente.

\section{c / Stade de lactation}

Le stade de lactation est un facteur de variation important des quantités ingérées par une vache laitière. Pour l'étudier, différentes prédictions ont été comparées (figure 4) à des données obtenues sur un groupe de vaches multipares ayant été alimentées avec le même régime tout au long de leur lactation (environ $70 \%$ ensilage de maïs et $30 \%$ aliments concentrés) (Vérité et al non publié). Les modèles de l'ARC et des Danois simulent l'évolution des quantités ingérées au cours de la lactation par une équation et des coefficients d'ajustement. Le système danois qui décrit bien le début de la lactation semble moins performant après. Les coefficients mensuels de l'ARC ont tendance à créer des paliers. Le modèle INRA ne propose une prédiction qu'après la fín du deuxième mois et, comme le système de Cornell, n'est doté d'aucun terme correctif pour le début de lactation. A ce titre, ils ne devraient pas figurer dans la comparaison pour les 2 premiers mois de lactation. Les courbes ont cependant été représentées entre crochets pour indiquer l'effet de l'absence de correction à ce stade (figure 4). La prédiction obtenue par ces 2 systèmes est mauvaise en début de lactation, mais elle montre que, passé la phase de mobilisation intense des réserves corporelles, les données de production et de poids vif suffisent pour décrire l'évolution des quantités ingérées. Enfin, le système du NRC utilise les variations de poids vif pour prédire les quantités ingérées mais ne propose aucune équation pour prédire l'intensité de cette mobilisation en fonction du stade de l'animal et de son niveau de production. Cette variable de variation de poids ayant une importance énorme dans le résultat de la prédiction, il est capital de prédire correctement l'intensité de la mobilisation. De plus, la mobilisation peut être elle-même dépendante de l'évolution des quantités ingérées et il est alors nécessaire d'utiliser un modèle dynamique comme l'a proposé
Forbes en 1977. Les calculs sont alors plus complexes, mais c'est sans doute le prix à payer pour améliorer la qualité des prédictions en début de lactation.

Le cas particulier de la vache laitière tarie met en défaut de nombreux systèmes qui ne

Figure 3. Simulation de l'effet de la production laitière des vaches sur la prédiction des quantités ingérées d'une ration complète composée d'ensilage de maïs (70\%) et d'aliments concentrés (30\%)

matière sèche ingérée $(\mathrm{kg} / \mathrm{j})$

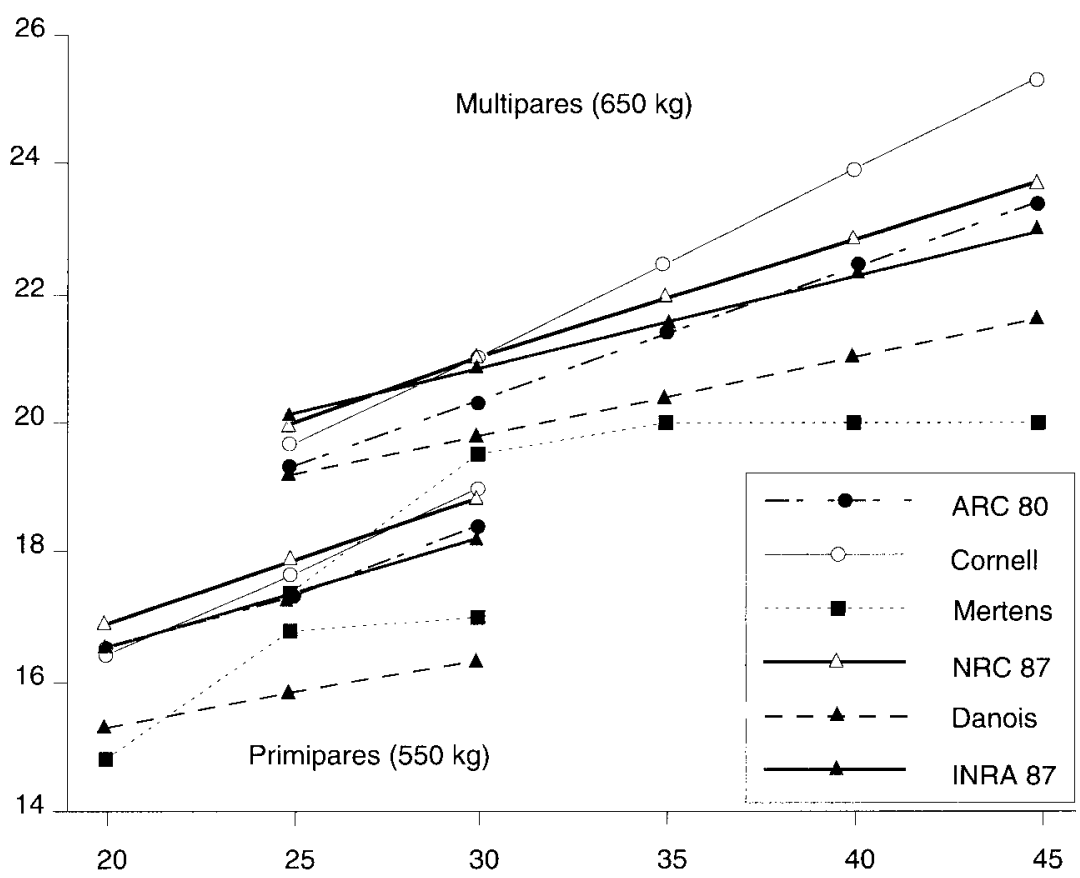

production de lait $4 \%(\mathrm{~kg} / \mathrm{j})$

Figure 4. Simulation de l'effet du stade de lactation des vaches laitières sur la prédiction des quantités ingérées d'une ration complète composée d'ensilage de maïs (70\%) et d'aliments concentrés $(30 \%)$. L'évolution des consommations observées est représentée à partir de données expérimentales (Vérité et al non publié).

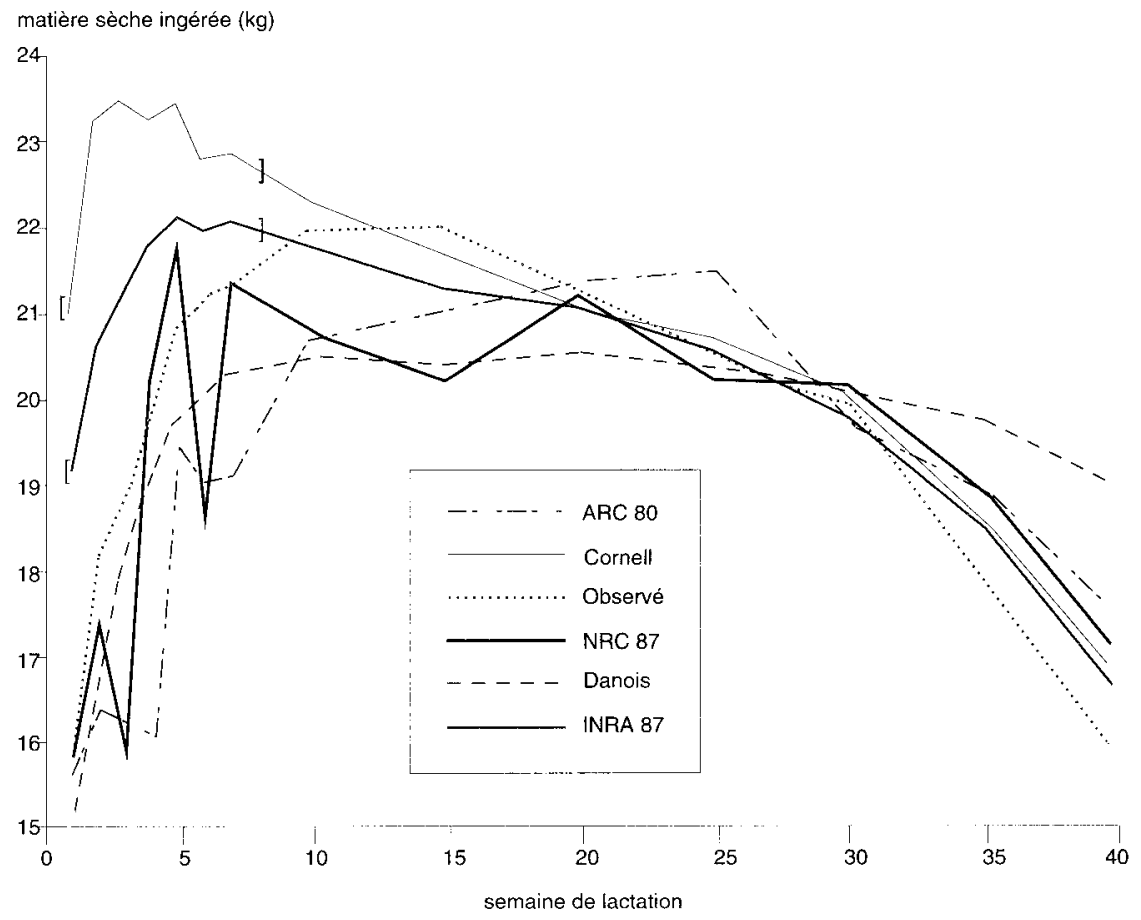


Tableau 2. Prédiction des quantités de matière sèche ingérée (kg/j) par les différents systèmes dans 2 situations différentes : (1) ration complète ensilage de maïs (70\%) aliments concentrés (30\%), production $35 \mathrm{~kg}$ de lait/j, (2) raygrass anglais 1er cycle avant épiaison au pâturage, production $25 \mathrm{~kg}$ de laitj.

\begin{tabular}{|l|c|c|}
\hline Référence & $\begin{array}{c}\text { Ration } \\
\text { complète (1) }\end{array}$ & Pâturage (2) \\
\hline MAFF (1975) & 19,0 & 17,0 \\
Neal et al (1984) & 20,6 & 17,7 \\
Journet et al (1965) & 20,5 & 17,5 \\
ARC (1980) & 20,6 & 18,6 \\
Bines (1979) & 20,2 & 19,5 \\
Mc Cullough (1981) & 23,0 & 18,1 \\
Mc Cullough (1973) & 22,1 & 18,7 \\
Vadiveloo et al (1979) & 19,7 & 14,5 \\
Yungblut et al (1981) & 22,6 & 17,6 \\
& & 16,2 \\
Greenhalgh et al (1966) & - & 18,5 \\
Curran et Holmes (1970) & - & 17,9 \\
Meijs et Hokstra (1984) & - & 11,9 \\
CSIRO (1990) & 20,4 & 17,5 \\
Brown et al (1977) & 22,1 & 18,1 \\
Cornell-Fox et al $(1990)$ & 19,2 & 17,9 \\
Forbes (1977) & 19,4 & 12,8 \\
Mertens (1987) & 20,8 & 19,5 \\
NRC (1987) & 16,8 & 12,8 \\
Hyppölä et Hasunen (1970) & 21,7 & 16,7 \\
INRA - Dulphy et al $(1987)$ & 20,4 & 16,7 \\
Kristensen et Ingvartsen (1986) & 21,9 & 18,3 \\
Valeurs observées & & \\
\hline
\end{tabular}

proposent pas d'équations adaptées. Le système INRA n'a pas proposé d'équation simplifiée pour le calcul des taux de substitution chez l'animal tari sans utiliser le modèle complet avec INRAtion (ce n'est pas très important à ce stade car l'apport d'aliments concentrés est généralement limité pendant cette période). Cependant, l'équation de prédiction de la CI semble valide pour les vaches taries simplement en utilisant une production de lait nulle. L'extrapolation des autres équations pour cette période conduit à des prédictions comprises entre 5 et $17 \mathrm{~kg}$ MS alors que les observations avec ce type de régime se situent entre 13 et $16 \mathrm{~kg}$ MS. La simulation des quantités ingérées au cours du cycle gestation-lactation peut et doit encore être améliorée, mais elle ne le sera qu'après une meilleure compréhension des mécanismes qui contrôlent l'évolution de la capacité d'ingestion durant ce cycle.

\section{2 / Ration}

\section{a / Fourrage}

Le système des unités d'encombrement permet une grande souplesse dans l'attribution des valeurs de l'encombrement d'un fourrage en se basant sur des mesures d'ingestibilité par rapport à un aliment de référence (l'herbe verte offerte à l'auge). La limite de cette méthode in vivo est qu'elle est peu pratique pour la détermination des valeurs d'encombrement d'aliments nouveaux ou obtenus dans des conditions climatiques particulières. De nombreuses équations ont été élaborées pour prédire des ingestibilités à partir des paramètres de la composition chimique des fourrages, mais elles sont sans doute trop nombreuses pour faciliter la tâche des utilisateurs. Les systèmes (Conrad, NRC, Forbes, Mertens) qui ne prennent en compte que la valeur en parois des fourrages ou leur fraction indigestible sont plus simples mais également beaucoup plus limités car ce critère n'explique qu'une partie des différences d'ingestibilité qui existent entre fourrages. Toutes les caractéristiques de palatabilité des aliments qui sont implicitement prises en compte par une mesure d'ingestibilité ne peuvent être intégrées dans une estimation de l'indigestible. Enfin, de nombreuses équations n'intègrent aucune caractéristique du fourrage et ne peuvent donc prédire correctement les quantités ingérées qu'à partir des paramètres de production de animaux observés a posteriori, ce qui limite l'intérêt de la prédiction.

En dehors de ces considérations liées à la méthode de prédiction utilisée, la comparaison des effets nature et qualité du fourrage entre les différents systèmes est très difficile à réaliser. Les conditions agro-climatiques diffèrent d'un pays à l'autre, conduisant à des caractéristiques des fourrages qui peuvent être propres à chaque situation. A titre d'illustration, le tableau 2 fournit des exemples de prédiction de quantités ingérées dans deux situations types que l'on peut rencontrer en France:

- un groupe de vaches laitières adultes produisant $3 \mathrm{~kg}$ de lait en dixième semaine de lactation et qui reçoivent à volonté un ration complète composée d'ensilage de maïs de bonne qualité $(70 \%)$ et d'aliments concentrés $(30 \%)$. La valeur proposée en référence provient d'essais conduit à la station de recherches sur la vache laitière (Vérité et $a l$, non publiés).

- un groupe de vaches laitières adultes produisant en moyenne $25 \mathrm{~kg}$ de lait en trentième semaine de lactation recevant au pâturage un Ray-Grass anglais ( ${ }^{\text {er }}$ cycle avant épiaison) offert à raison de $30 \mathrm{~kg} \mathrm{MO} / \mathrm{vache} / \mathrm{j}$ sans aucune complémentation énergétique. La valeur de référence provient d'un travail où les quantités ingérées ont été mesurées à plusieurs reprises au pâturage par la méthode des index fécaux (Comeron 1991).

Avec le régime à base d'ensilage de maïs, les systèmes britanniques et danois tendent à sous-estimer les quantités ingérées observées dans nos conditions, alors que les systèmes français et américain sont globalement plus proches (sauf Mertens). L'utilisation d'un ensilage de maïs riche en grain dans les conditions anglaises ou danoises n'est sans doute pas courante et explique pour partie les écarts observés. Avec le pâturage, les prédictions des systèmes basés sur les caractéristiques de besoins des animaux sont plus proches des mesures effectuées que celles des systèmes qui utilisent les caractéristiques de l'herbe et les notions d'encombrement, mais ce sont des prédictions a posteriori. Ceci reflète toute la difficulté qu'il 
y a à prédire l'utilisation de l'herbe lorsqu'elle est sur pied, avec toutes les contraintes et les possibilités de choix auxquelles est soumis l'animal au pâturage.

\section{b / Apport d'aliments concentrés}

Pour qu'un système soit capable de répondre à des modifications de la composition de ration, il faut que ses équations utilisent des variables descriptives de cette composition. Dans le cas contraire, la prédiction restera insensible à la composition de la ration. Pour illustrer l'effet de l'apport d'aliments concentrés, les différents systèmes ont été utilisés pour prédire la situation expérimentale décrite par Ostergaard (1979) avec des vaches recevant un ensilage d'herbe (figure 4). Les paramètres de la ration, quand ils existent, ont été choisis pour que la prédiction de l'ingestion de fourrage seul soit identique dans tous les systèmes de manière à ne pas cumuler le problème de la prédiction de l'ingestibilité du fourrage. L'équation de l'ARC, comme toute équation qui n'intègre pas les paramètres aliments, reste constante. Si l'équation prédit un coefficient de substitution constant, l'ingestion s'accroît linéairement quel que soit l'apport d'aliments concentrés (Vadiveloo et al 1979, Danois). Si le système utilise 2 équations, l'une énergétique l'autre physique (NRC), la MST prédite passe par un maximum avant de diminuer, ce qui revient à utiliser un taux de substitution qui passerait brutalement de 0,5 à 1,2. Enfin, si le système de prédiction intègre un taux de substitution qui s'accroît progressivement avec l'apport d'aliments concentrés (INRA ou Lewis), la MST prédite suit une courbe parabolique semblable à celle décrite par Ostergaard (figure 5). Les différences de prédiction obtenues entre les systèmes peuvent paraître limitées ( 1 à $2 \mathrm{~kg}$ MS, sauf ARC) mais elles peuvent conditionner de façon

Figure 6. Corrections de l'ingestion attendue sous l'effet des conditions climatiques (d'après NRC 1987).

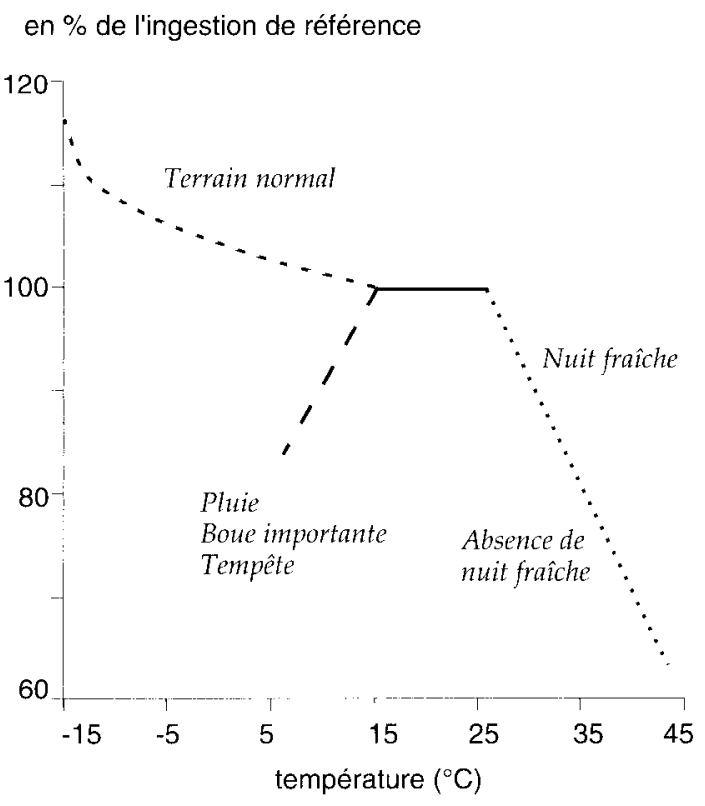

Figure 5. Simulation de l'effet du niveau d'apport d'aliments concentrés en complément d'un ensilage d'herbe sur la prédiction des quantités ingérées. L'évolution des consommations observées est représentée à partir des résultats expérimentaux d'Ostergaard (1979).

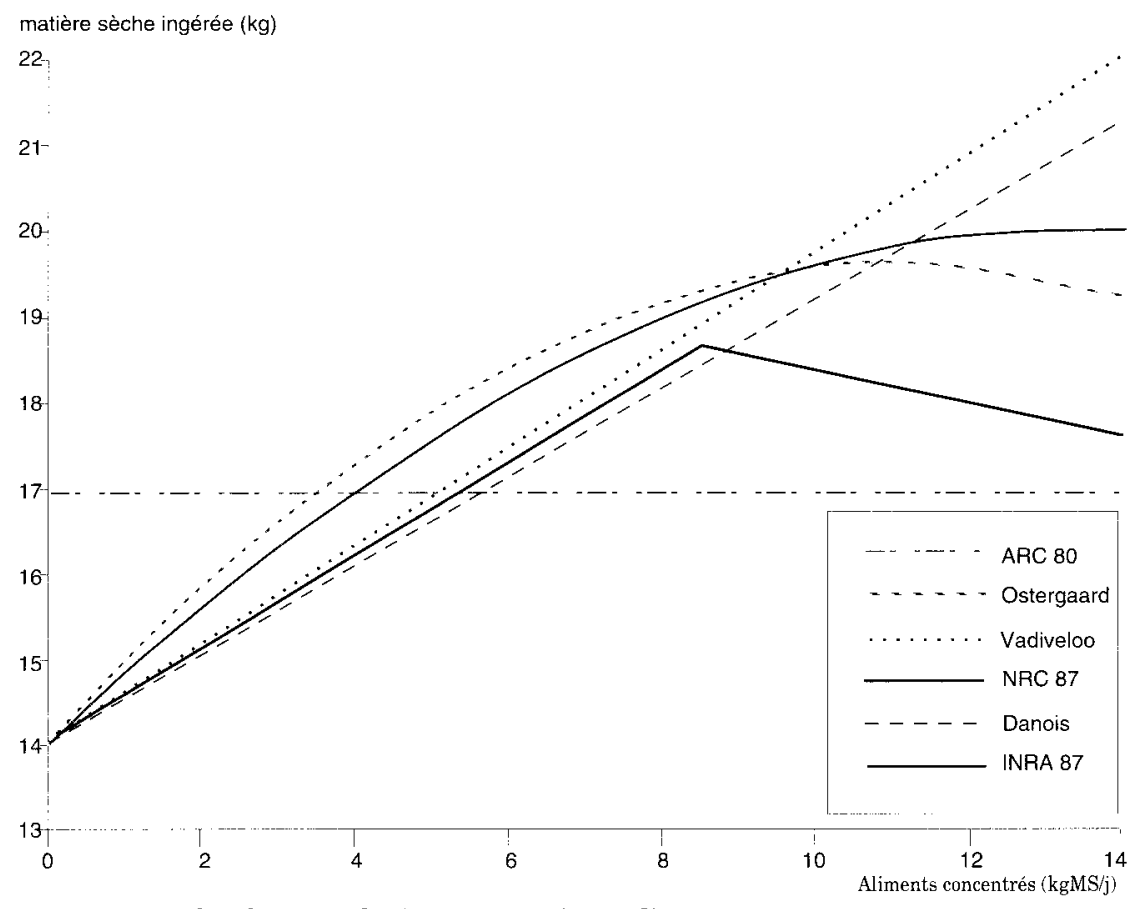

importante le choix technico-économique d'une stratégie de complémentation en aliment concentré.

\section{3 / Environnement}

Peu de systèmes essayent de prendre en compte les facteurs liés à l'environnement. Ceci peut s'expliquer par le fait que ces méthodes de prévision de l'ingestion sont essentiellement destinées à établir des rationnements hivernaux de vaches en stabulation et dans des climats relativement tempérés, ce qui ne nécessite pas toujours la prise en compte des facteurs climatiques. Seul le système du NRC propose des recommandations liées à l'environnement sous forme de facteur correctif multiplicateur (figure 6). Il en va tout autrement pour les systèmes de prédiction des quantités ingérées au pâturage et il est étonnant que seul le système australien propose des facteurs correctifs.

\section{3 / Les applications des différentes méthodes aux pratiques de rationnement}

Les équations de prédiction des quantités ingérées sont établies à partir de données expérimentales, elles sont donc liées aux pratiques de rationnement utilisées. Le nombre et la diversité de ces données d'une part, le concept de la méthode de prédiction d'autre part constituent des éléments qui permettent de définir les limites d'application des systèmes de prédiction de quantités ingérées. 


\section{1 / Adaptation à des régimes différents}

Compte tenu de la diversité des rations utilisables en France, il est indispensable qu'un système de prédiction des quantités ingérées puisse intégrer correctement la diversité des régimes. Toutes les équations qui n'intègrent pas les caractéristiques de la ration posent un problème majeur pour décrire cette diversité, sauf si la complémentation en aliments concentrés vient rééquilibrer les disparités entre fourrages. Il est par exemple intéressant de noter qu'aucune équation ne prend en compte la structure physique d'un fourrage ou la qualité de conservation d'un ensilage alors que ces données modifient notablement l'ingestion. Le système des UE peut sans problème intégrer ces différences (en particulier pour les ensilages d'herbe).

Les pratiques de distribution des aliments sont également différentes puisque le concentré peut être distribué séparément ou mélangé dans une ration unique, ou les deux. La plupart des méthodes de calcul ne sont prévues que pour une méthode de distribution : pour les rations complètes (systèmes américains) les équations utilisent le pourcentage d'aliments concentrés (CR), alors que pour la distribution séparée du concentré (systèmes européen) c'est la quantité d'aliments concentré (C). Il est parfois mathématiquement possible de passer de la quantité d'aliment concentré à un pourcentage mais cette transposition n'est pas simple. Les systèmes basés sur les UE doivent théoriquement pouvoir calculer dans les 2 unités. C'est très simple avec le système danois, car il ne prend pas en compte les variations de l'encombrement de l'aliment concentré, les phénomènes de substitution étant supposés constants. Le système français des UE a récemment adapté le modèle du taux de substitution au cas des rations complètes et permet actuellement d'effectuer ces calculs avec le logiciel INRAtion. A l'avenir, des tables ou des équations simplifiées sont envisageables pour une utilisation plus pratique.

Enfin, il existe une littérature beaucoup plus abondante sur la prédiction des quantités ingérées à l'auge qu'au pâturage. Ceci s'explique par la difficulté d'estimer les quantités ingérées au pâturage. Les vaches n'en mangent pas moins de l'herbe pendant de longues périodes de l'année. Le système français des UE, comme les autres systèmes UE, donne des prédictions qui peuvent sous-estimer l'ingestion d'herbe au pâturage lorsque l'herbe est de bonne qualité et allouée en quantité suffisante (tableau 2), sans doute en raison des possibilités de tri. Lorsque le problème d'une complémentation se pose (biomasse insuffisante, chargement trop élevé, qualité médiocre de la pâture) les équations semblent ne plus fonctionner aussi valablement. Les systèmes qui utilisent les données de production et de variation de poids pour estimer les quantités ingérées sont assez proches des données observées (Forbes, Cornell, Bines,...). Cependant ils ne sont corrects qu'a posteriori car ils ne permettent pas de prédire les variations de besoin de l'animal. Meijs et Hoekstra (1984) et Caird et Holmes (1986) ont introduit des variables descriptives de la pâture pour prendre en compte ces facteurs, mais parfois au détriment des caractéristiques de l'animal (Meijs et Hoekstra, 1984). La précision obtenue permet de penser qu'un travail important reste à accomplir dans ce secteur. Les australiens (CSIRO 1990) ont introduit des éléments de modélisation du comportement alimentaire au pâturage pour prédire les quantités ingérées par les moutons (Christian et al 1978) mais ne semblent pas l'avoir étendu pour l'instant aux vaches laitières.

\section{2 / Recommandation alimentaire ou prédiction des quantités ingérées?}

Les équations de prédiction ont été établies à partir de données expérimentales obtenues dans des conditions qui sont généralement spécifiques de chaque pays. De ce fait, elles sont presque toujours très dépendantes des aliments distribués, des races d'animaux et des méthodes de rationnement utilisées. Lorsque les équations ont été établies à partir de rations riches en concentrés, ces équations seront souvent peu précises avec des régimes riches en fourrages et inversement. Les animaux de races laitières britanniques semblent avoir assez systématiquement des capacités d'ingestion plus faibles que les races pie-noires françaises ce qui peut expliquer les différences de prédiction observées dans certaines situations. La plupart de ces systèmes ne cherchent donc pas à prédire les quantités ingérées dans toutes les situations possibles mais permettent de réaliser des calculs de rationnement dans des conditions assez stéréotypées. Ce sont donc plus des outils de rationnement que des systèmes de prédiction des quantités ingérées. Aussi, l'utilisation d'un système élaboré dans des conditions différentes de celles d'application devra faire l'objet d'une validation approfondie au préalable.

Le système des UE permet de prédire, comme le modèle plus complexe de Forbes (1977), le bilan énergétique d'un animal recevant une nouvelle ration. En utilisant les équations sur la réponse marginale de la production laitière en fonction du bilan énergétique (Coulon et Rémond 1991), il est alors possible de prédire les conséquences d'une sous(sur)-alimentation sur la production et donc sur la capacité d'ingestion. Cette approche est très différente de celle des équations qui intègrent le résultat d'un rationnement (production et variation de poids vif) pour prédire les quantités ingérées en effectuant ainsi une "prédiction a posteriori". La plupart des régressions multiples obtenues sur des données expérimentales fonctionnent ainsi mais ne permettent en pratique de prédire les quantités ingérées que si les besoins sont satisfaits. 
Le système de UE est sans doute un des plus performants dans son aptitude à prendre en compte une grande diversité de rations, $\mathrm{y}$ compris lorsque les apports énergétiques diffèrent des besoins. Ceci permet à l'éleveur de choisir l'objectif de rationnement qui s'adapte le mieux à son environnement technique et économique, et lui évite de subir la recommandation unique d'un système de rationnement qui ne tient pas compte des contraintes de l'éleveur.

\section{3 / Aptitude à évoluer, portabilité du système}

Suivant les méthodes utilisées, les systèmes de prédiction des quantités ingérées pourront intégrer de nouveaux aliments, de nouvelles technologies, l'effet du progrès génétique s'ils sont évolutifs ou seront condamnés à disparaître si leur structure est figée. Une régression multiple ne peut évoluer, elle change. Les systèmes basés sur les UE peuvent évoluer. Il est très intéressant de pouvoir conserver une démarche de calcul et de rationnement tout en améliorant la qualité de la prédiction. L'utilisateur pourra ainsi intégrer l'évolution des concepts en utilisant la même méthode pour ses calculs de rations.

De plus, les techniciens de l'alimentation animale sont amenés à travailler sur plusieurs espèces simultanément. Un système dont les principes sont communs à toutes les espèces de ruminants offre les mêmes avantages qu'un environnement commun à tous les logiciels présents sur un même ordinateur et qui permet d'échanger des données. Le système des
UE est sans conteste celui qui est allé le plus loin dans cette direction. Cette ossature commune devrait, de plus, constituer un atout pédagogique intéressant pour l'enseignement.

\section{Conclusion}

Aucun système n'est à l'heure actuelle capable de décrire correctement tous les grands facteurs de variations des quantités ingérées connus. La comparaison des différents systèmes permet de mieux dégager les grandes lignes de ce que devrait être l'évolution du système INRA des UE pour les vaches laitières. L'amélioration de la prédiction de la capacité d'ingestion est essentielle, en particulier en début de lactation. Celle-ci ne pourra se faire valablement qu'en intégrant les mécanismes de la gestion des réserves corporelles dans la régulation de la capacité d'ingestion au cours du cycle gestation-lactation. Au niveau de la ration, la séparation parfois délicate entre fourrages et aliments concentrés risque de poser de plus en plus de problèmes à l'avenir. Pour lever cette difficulté, il n'est pas impossible qu'il faille repenser la méthode de prédiction des valeurs d'encombrement en conservant les qualités du système actuel. Malgré ces limites, le système INRA représente cependant un compromis équilibré entre complexité et performances, capable de décrire aussi bien les facteurs de variation de la ration que ceux de l'animal. Son aptitude à évoluer et son adaptation aux différentes espèces de ruminants sont des atouts importants pour son développement.

\section{Références bibliographiques}

A.R.C., 1980. Feed Intake. In: The nutrient requirements of ruminant livestock. Commonwealth Agricultural Bureaux, Furnham Royal, Slough, England, 59-72.

Bines J.A., 1979. Voluntary Food Intake. In: H. Swan and W.H. Brostier (Editors), Feeding strategy for the high yielding dairy cow. London, Granada, 23-48.

Brown C.A., Chandler P.T., Holter J.B., 1977. Development of predictive equations for milk yield and dry matter intake in lactating cows. J. Dairy Sci., 60: 1739-1754.

Caird L., Holmes W., 1986. The prediction of voluntary intake of grazing dairy cows. J. Agric. Sci. Camb., 107: 43-54.

Christian K.R., Freer M., Donnelly J.R., Davidson J.L., Armstrong J.S., 1978. Simulation of grazing systems. (Centre for Agricultural Publishing and Documentation : Wageningen).

Comeron E. A., 1991. Estimation des quantités ingérées par des vaches laitières au pâturage $: 1$ ) influence des caractéristiques de sanimaux, 2) influence de la quantité et de la structure de l'herbe offerte. Thèse Docteur Université Rennes 1. $156 \mathrm{p}$

Conrad H.R., Pratt A.D., Hibbs, J.W., 1964. Regulation of feed intake in dairy cows. 1. Change in importance of physical and physiological factors with increasing digestibility. J. Dairy Sci., 47: 54-62.

Coulon J.B., Rémond B., 1991. Réponses de la production et de la composition du lait de vache aux variations d'apports nutritifs. INRA Prod. Anim., 4 (1): 49-56.

CSIRO, 1990. Feeding standards for Australian livestock Ruminants. J. Corbett Ed., CSIRO Australia, 288 p.
Curran, M.K. and Holmes, W., 1970. Prediction of the voluntary intake of food by dairy cows. 2 - Lactating grazing cows. Anim. Prod., 12: 213-224.

Curran, M.K., Wimble, R.H. and Holmes, W., 1970. Prediction of the voluntary intake of food by dairy cows. 1. Stall-fed cows in late pregnancy and early lactation. Anim. Prod., 12: 195-212.

Demarquilly C., Andrieu J., Sauvant D., Dulphy J.P., 1978. Composition et valeur nutritive des aliments. In "Alimentation des ruminants", INRA Publications, Versailles, France, 597 p.

Dulphy J.P., Faverdin P., 1987. Lingestion alimentaire chez les ruminants : modalités et phénomènes digestifs associés. Reprod. Nutr. Dévelop., 27 (1B): 129-155.

Dulphy J.P., Faverdin P., Micol D., Bocquier, F., 1987. Révision du système des unités d'encombrement (UE). Bull. Tech. CRZV Theix, INRA, 70: 35-48.

Faverdin P., Hoden A., Coulon J.B., 1987. Recommandations alimentaires pour les vaches laitières. Bull. Tech. C.R.Z.V. Theix, INRA, 70: 133-152.

Faverdin P., Dulphy J.P., Coulon J.B., Vérité R., Garel J.P., Rouel J., Marquis B., 1992. Les phénomènes de substitution fourrages -concentrés chez la vache laitière. INRA Prod. Anim., 5(2): 127-135.

Forbes J.M., 1977. Development of a model of voluntary food intake and energy balance in lactating cows. Anim. Prod. 24 : 203-224.

Forbes J.M., 1983. Models for the prediction of food intake and energy balance in dairy cows. Livest. Prod. Sci., 10:149-157. 
Forbes J.M., 1986. The voluntary food intake of farm animals. Butterworths, London, $206 \mathrm{pp}$.

Fox D.G., Sniffen C.J., O'Connor J.D., Russell J.B., Van Soest P.J., 1990. The Cornell net carbohydrate and protein system for evaluating cattle diets. Cornell Univ. Agric. Exp. Sta. $\mathrm{N}^{\circ} 34$, Ithaca.

Greenhalgh J.F.D., Reid G.W., Aitken J.N., Florence E., 1966 The effects of grazing intensity on herbage consumption and animal production: 1 . Short-term effects in strip-grazed dairy cows. J. Agric. Sci. Camb., 67: 13-23.

Hyppola K., Hasunen O., 1970. Dry matter and energy standards for dairy cows. Acta Agral. Fenn. 116: 1-41.

Jarrige R., 1986. Voluntary intake in dairy cows and its prediction. In : C.C. Balch and A.J.M. Van Es (Editors), Recent advances in feed evaluation and rationning systems for dairy cattle in extensive and intensive production systems. Int. Dairy Fed. Bull. 196: 4-16.

Jarrige R., Demarquilly C., Dulphy J.P., Hoden A., Journet M. Béranger C., Geay Y., Malterre C., Micol D., Petit M., Robelin J., 1979. Le système des unités d'encombrement pour les bovins. Bull. Tech. C.R.Z.V. Theix, INRA, 38: 57-79.

Journet M., Poutous M., Calomiti S., 1965. Appétit de la vache laitière. 1. Variations individuelles des quantités ingérées. Ann. Zootech., 14: 5-37.

Kristensen V.F., Ingvartsen K.L., 1986. Prediction of feed intake, In: "New developments and future perspectives in research on rumen function". Neimann - Sorensen A. (Ed). Commission of the European communities, Luxembourg, 157181.

Lewis M., 1981. Equations for predicting silage intake by beef and dairy cattle. Proceedings of the sixth silage conference, Edinburgh, 1981, 35-36.

MAFF, 1975. Energy allowaces and feeding systems for ruminants. Tech. Bull. 33, Ministry of Agriculture and Fisheries, London.
Mc Cullough M.E., 1973. cité par : Mc Cullough M.E., 1983. Predicting dry matter intake in ruminants. Proc. Georgia Nutr. Conf. Feed. Ind. 1983, p. 45-48

McCullough M.E., 1981. Feed programming of optimum rations for milk production and growth. Misc. Pub. $110 \mathrm{Ga}$. Cooperative extension service, Athens, GA

Meijs J.A., Hoekstra J.A., 1984. Concentrate supplementation of grazing dairy cows. 1. Effect of concentrate intake and herbage allowance on herbage intake. Grass Forage Sci. 39: 59-66.

Mertens D.R., 1987. Prediction intake and digestibility using mathematical models of ruminal function. J. Anim. Sci., 64 1548-1558.

Neal H.D.St.C., Thomas C., Cobby J.M., 1984. Comparisons of equations for predicting voluntary intake by dairy cows. $\mathrm{J}$ Agric. Sci. Camb., 103: 1-10.

N.R.C., 1987. Predicting Feed intake of food-producing animals. National Academy Press, Washington, $82 \mathrm{p}$.

Ostergaard V., 1979. Strategies for concentrate feeding to attain optimum feeding level in high yielding cows. Rep.482, Natl. Inst. Anim. Sci., Copenhagen, $138 \mathrm{p}$

Owdongo W.O., Conrad H.R., 1983. Prediction of digestible and net energy intake in lactating dairy cows. J. Dairy Sci., 66: 166 (Abstr.)

Vadiveloo J., Holmes W., 1979. The prediction of the voluntary feed intake of dairy cows. J. Agric. Sci. Camb., 93: 553-562

Waldo D.R., 1986. Effect of forage quality on intake and forage concentrate interactions. J. Dairy Sci., 69: 617-631.

Wangsness P.J., Muller L.D., 1981. Maximum forage for dairy cows: review. J. Dairy Sci., 64: 1-13.

Yungblut D.H., Stone J.B., Mcleod G.K., Grieve D.G., Burnside E.B., 1981. The development of feed intake prediction equations for lactating dairy cows. Can. J. Anim. Sci., 61: 151-157.

\section{Summary}

Dairy cow feeding : comparison of the different methods of prediction of dry matter intake.

The different methods of predicting the dry matter intake can be grouped into 3 main types:

1 / multiple regression equations, generally empirical and produced from experimental data,

2 / the system of two predictions, one linked to a physical regulation of intake (rumen fill), the other to an energetic regulation (energy balance) of which only the lesser value is conserved for the prediction of dry matter intake,

$3 /$ the system based on Fill Unit of roughages and the substitution of concentrates to roughages.

The comparison of these different systems shows that the factors which are the most difficult to take into account remain those of the diversity of feed and their respective proportions in the ration, together with that of the beginning of lactation.
The majority of these systems are more for establi. shing optimum ration calculations of feed recommen. dations rather than really predicting ingested quantities, taking into account all the possible factors of variation. They are closely linked to feeding practices (complete diet rich in by-products and concentrates in the U.S.A., individual adjustments in concentrate supply with rations composed mainly of roughages in Europe). The Fill Unit system proposed by INRA does have certain limits in the prediction of the fill value of feed (separation of roughages and concentrates, numerous equations) and in its use at the beginning of lactation. At the present time, this is the tool which enables the greatest diversity of situations to be described, whilst conserving a calculation method which is easy to use in practice and which has possibilities of being developed.

FAVERDIN P., 1992. Alimentation des vaches laitières : comparaison des différentes méthodes de prédiction des quantités ingérées. INRA Prod. Anim., 5 (4), 271-282. 